\title{
Authors response to comments on article in Natural Hazards (2008) NHAZ 524, Article 9299, DOI 10.1007/s11069-008-9299-2
}

\author{
Mansi Daga $\cdot$ M. C. Deo
}

Received: 2 March 2009/Accepted: 15 April 2009/Published online: 3 May 2009

(C) Springer Science+Business Media B.V. 2009

We thank the discussers for their comments. Our objective in this article was to evaluate the performance of different data-driven methods, including genetic programming (GP) in carrying out inverse modeling tasks. We tried to explain the basic idea of GP in general terms. While the code used by us was based on a variant of GP, as pointed out by the discussers, the extension of genetic algorithm to genetic programming can always be explained in general terms for the benefit of unfamiliar readers, as done by us.

We, however, agree with the discusser in that there exists a scope to take the lead of applying GP further from this study, and this may include studying if team solutions can yield better performance and so on. 